\title{
Analogy Methods to Address Warping and Plasticity in Torsion
}

\section{Prof. Somnath Chattopadhyay, University at Buffalo, SUNY}

Dr. Somnath Chattopadhyay teaches mechanics, materials, manufacturing and design at University at Buffalo He has authored a text on Pressure Vessel s and till recently was an Associate Editor of the ASME Journal of Pressure Vessel Technology. His research interests are in the areas of fatigue and fracture of metals, carbon nanotubes, multi-scale material modeling and engineering education. He had a very successful industrial career with Westinghouse Electric where he directed and performed structural design of power generation equipment and is a registered professional engineer. He received his Ph.D. in mechanics from Princeton University. 


\title{
Analogy Methods to Address Warping and Plasticity in Torsion
}

\author{
ABSTRACT \\ This project is primarily an experimental study on the membrane analogy and the sand-heap \\ analogy associated with plasticity (along with warping) when a prismatic bar (circular or non- \\ circular) is twisted. This is a supplemental lab activity for the junior level Mechanics of \\ Materials course. When a prismatic bar of arbitrary cross-section is twisted, it gets deformed into \\ helical curves as the cross sections rotate during torsion. The analogy between the elastic torsion \\ of a bar and the small deflection of a laterally loaded membrane is what is known as the \\ membrane analogy. The membrane is stretched by a uniform tension and is attached to a die \\ whose edge plane is the same as the cross section of the twisted bar. A uniform lateral pressure is \\ applied to the membrane to produce a deflection. Except for a circular cylindrical bar, the cross- \\ sections become warped and it plays a significant role in structural design. As the torque is \\ increased, the material passes from the elastic to plastic state. There is a limit to the ability of the \\ cross section to withstand increasing torque. This feature is not typically addressed in the junior \\ level Mechanics of Materials course, but we felt the need for it because this deformation can be \\ readily shown in a torsion experiment with a ductile material such as aluminum. When the torque \\ is increased to a critical value, the resultant shear stress attains the yield value ' $k$ ' at one or more \\ points, somewhere along the boundary of the cross section. The extension of the membrane \\ analogy to elastic plastic torsion leads to the sand-heap analogy. A sand heap is formed on a base \\ having a cross-section similar to the cross-section under investigation. In the case of membrane \\ analogy, the volume bounded by the deflected membrane is proportional to the applied torque. \\ For the sand heap analogy, the volume of the sand heap is proportional to the limiting torque \\ applied to the specimen. Experimental estimates for the elastic and inelastic torques in twisting
}


circular and non-circular shafts are in reasonable agreement with the values predicted by the membrane analogy and the sand heap analogy.

\section{INTRODUCTION}

In this work, prismatic bars of circular and square sections have been loaded in torsion to study their responses in elastic and elastic-plastic domains. The purpose was to experimentally demonstrate the plastic deformation that takes place when the torque exceeds the amount that causes the bar to yield. This is an extension of the torsion test where the torques are such that yielding does not take place, and the torque twist characteristic is linear. In such tests typically bars of circular cross are used. Bars of square cross section were used in our work to experimentally demonstrate the phenomenon of warping that takes place for bars of non-circular sections under torsion. When a bar is twisted beyond the elastic range (yielding) there is a non-linear torque-twist behavior. Specifically, for bars of non-circular cross-section it is hard to separate the warping deformation from the plastic deformation. Simple analytical solutions for the deformation and stresses in a bar subject to axial torsion exist only for circular cross-sections. However, it is sometimes necessary to design shafts of noncircular cross-sections. As an example, the design of a connecting rod would require determination of torsional stresses in non-circular sections (typically I-sections). Such situations also exist in the design of various machine parts such as brackets and supports that are sometimes loaded in torsion. The determination of the stresses and deflections for the torsion of non-circular shafts involve equations that are quite complicated. The assumptions that are valid for circular cross-sections do not apply here. For non-circular shafts under torsion, the plane cross-sections perpendicular to the shaft axis do not remain plane after twisting, and deformation takes place in the axial direction which is called warping. 
One of the simplest ways to study elastic as well as elastic-plastic behavior analytically is to assume an elastic-perfectly plastic representation of the stress-strain curve. For the case of torsion this would be the shear stress vs. shear strain curve as shown in Figure 1. The shear stress varies linearly with shear strain in the elastic region, and reaches a constant value and stays constant in the plastic region. Sometimes this representation is termed as one of "zero hardening." However, most ductile materials exhibit strain hardening where in the plastic region the shear stress monotonically increases with shear strain.

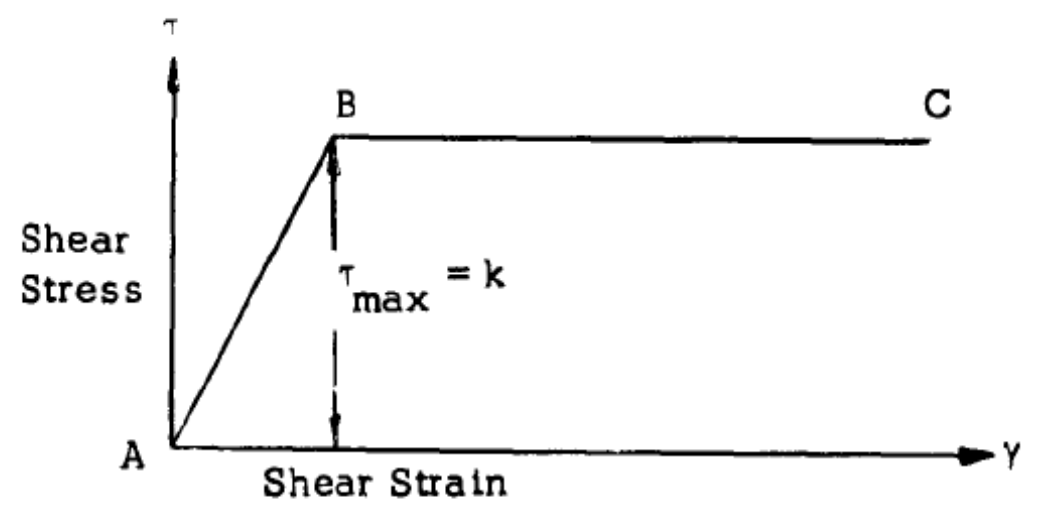

Figure 1 Idealized Shearing Stress-Strain Curve

The focus of this work is on analogy methods to study elastic and elastic-plastic torsion. The two analogies associated with torsion are described below.

\section{The Membrane Analogy for Elastic Torsion}

An analogy between the elastic torsion of a bar and the small deflection of a laterally loaded membrane has been pointed out by Prandtl. ${ }^{[1]}$ The membrane is stretched by a uniform tension $F$ per unit length of its boundary, and is attached to a die whose edge plane is of the same shape as 
the cross section of the twisted bar. A uniform lateral pressure is then applied to the membrane to produce a deflection $w$ at a generic point). The boundary condition is $w=0$ along the edge of

the die. It turns out that the contours of constant deflection correspond to the lines of shearing of the twisted bar. Also the applied torque is proportional to the volume bounded by the deflected membrane and the $x y$ plane. Since the membrane is everywhere concave to the applied pressure, the greatest value of the shear stress must occur somewhere on the boundary.

Consider a cylindrical or prismatic bar of constant cross section which is twisted and held in equilibrium by twisting moments applied at its ends. The bar is considered to be composed of an isotropic material possessing the idealized stress-strain relationship for an elastic, perfectly plastic material shown in Figure 1. Increasing torque causes the material to pass from the elastic region (line $\mathrm{AB}$, Figure 1) into the perfectly plastic range (line $\mathrm{BC}$, Figure 1). After a point in the cross section reaches the yield stress in shear (point B), this maximum shearing stress remains a constant value $\mathrm{k}$ as increasing torque causes an increase in the plastic region of the bar. Before examining the plastic behavior of the prismatic cross section, we will consider the stress characteristics in the elastic range

\section{The Sand-Heap Analogy for Plastic Torsion}

An extension of the membrane analogy to elastic/plastic torsion has also been suggested by Prandtl ${ }^{[1]}$ It is necessary to erect a roof of constant slope having its base similar to the boundary of the cross section.

If the bar of certain cross-section is twisted beyond the yield point, certain parts of the bar will be deformed plastically. Similar to the case of the elastic torsion, the shearing stresses are directed 
tangentially to the contour lines of the plastic stress surface. The plastic stress function may be considered as a "roof" under which the membrane, geometrically the same as the cross section, expands. When the membrane touches the "roof," the condition of plasticity is satisfied and plastic yielding begins at that point in the cross section. As the torque is increased, as represented by increasing air pressure on the membrane, the membrane expands and touches more of the "roof." At the limit the membrane fills the entire volume under the roof. The cross section is considered to have attained a fully plastic state. Following the membrane analogy, the torque required to achieve the fully plastic state is proportional to the volume under the "roof." The mathematical and physical interpretation of the plastic response for the case of complete yielding, of the entire prismatic bar can be demonstrated by sand heaps covering a plate similar in cross section to the twisted bar. This analogy was first presented by Nadai ${ }^{[2]}$ at a meeting of the German Society of Applied Mathematics and Mechanics in Marburg, Germany in 1923. ."A plate whose shape is geometrically similar to the cross section of the twisted bar series as a horizontal tray to hold a heap of dry uniform sand. The heap is to be as big as it is possible to pile by pouring a gentle stream of sand on top of the model, the excessive sand rolling freely down the slopes of the heap and falling off the elevated tray. ."

In a solid bar made of non-hardening material, the fully plastic stress distribution represents a limiting state which is approached in an asymptotic manner as the angle of twist increases. The fully plastic value of the torque has a physical significance, since it is very closely attained while the deformation is still of the elastic order of magnitude. The stress surface for a fully plastic cross section can be obtained experimentally by piling dry sand on a horizontal base whose shape is geometrically similar to that of the cross section of the bar. The limiting torque is 
proportional to the volume of sand forming the hill. Once the limiting torque has been reached, the bar is free to twist in an unrestricted manner.

Some results ${ }^{[3]}$ of the computation for a square cross section of side $2 a$, based are shown in Figure 2 . Choosing $n=9$, which very nearly represents a perfectly plastic material, the positions of the elastic/plastic boundary for increasing angles of twist are obtained as contours of $\tau=k$, and plotted in (a). The influence of work-hardening on the torque-twist relationship is indicated in $(b)$.

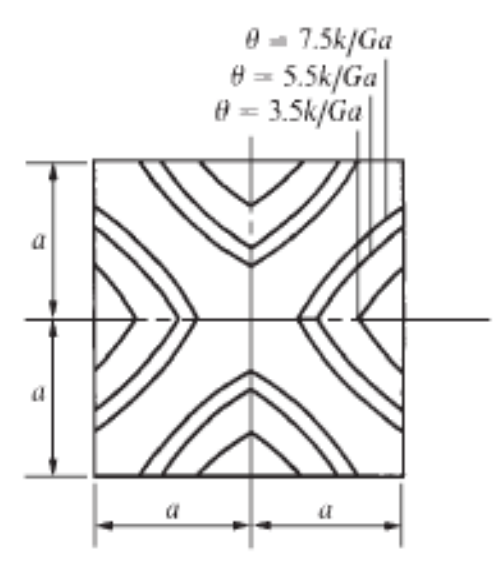

(a)

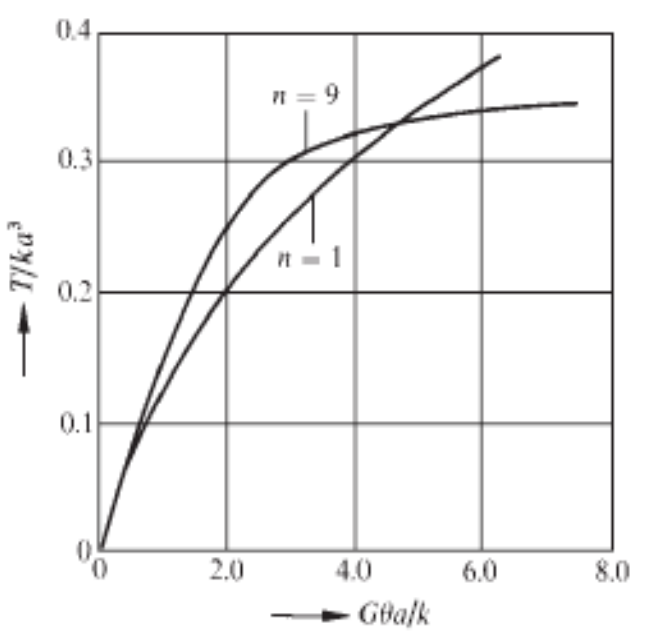

(b)

Figure 2: Numerical results for torsion of a square cross-section

\section{WARPING}

Warping was demonstrated by using the membrane analogy. A steel plate with a square hole was used. Rubber sheet was rigidly clamped at the edges of the hole and made to bulge by applying pressure from beneath the plate. The resulting bulges (torsional hills) for the square hole is 
shown in Figure 3.

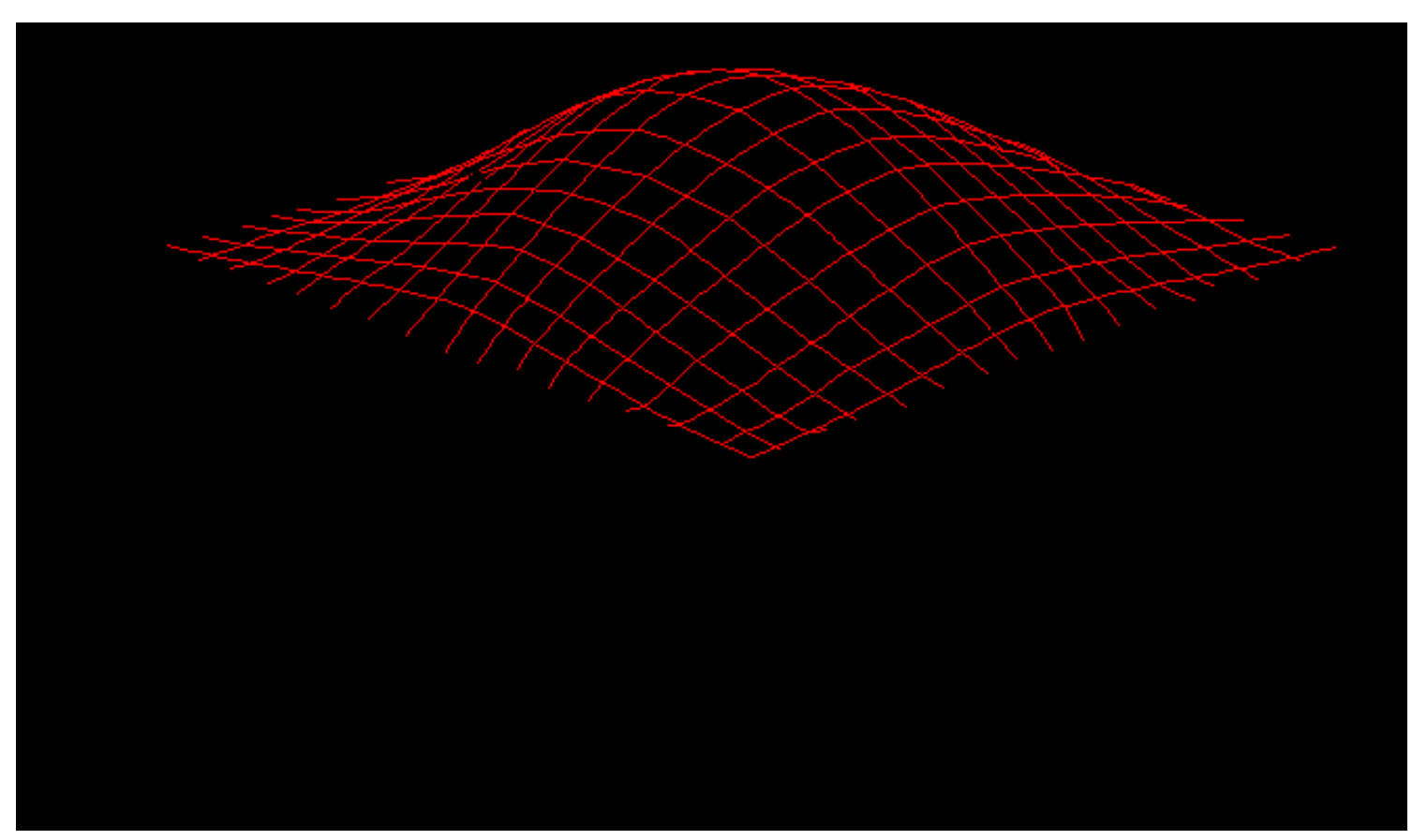

Figure 3 Torsion Hill for Square Cross-Section

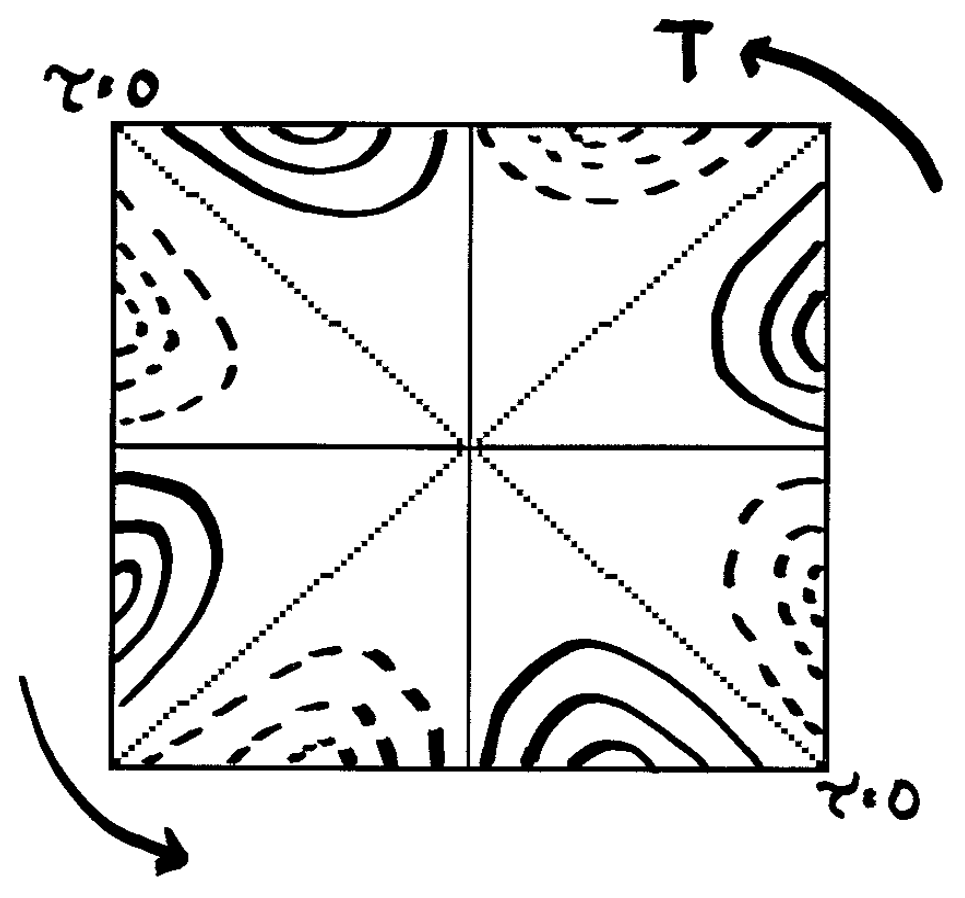

Figure 4 Shear Stress Distributions on a Square Shaft in Torsion 
For the square hole, the membrane will be forced to lie flat (zero slope) at the corners and steepest slopes at the midpoints of the outer edges.

The students are alerted to the fact that the analysis of a non-circular torsion member is far more complicated than a circular shaft. It will be wrong to assume that the shearing stress in the cross section of a square bar varies linearly with the distance from the axis of the bar, and thus is largest at the corners of the cross-section. The shear stress is actually zero at the corners. The distorted shape indicative of warping as shown in Figure 1 is due in part to the fact that shear stresses at the corners vanish. The shear stress distribution on a square shaft under torsion is shown in Figure 4.

The students are also made aware of the fact that the torsional shear stresses in a shaft of arbitrary cross-section are proportional to the slopes of a suitably inflated flexible membrane (membrane analogy). Thus the stress is a maximum at the midpoint of the outer edges of a square shaft, and not at the corners. In the corners the membrane has a zero slope indicating a zero stress.

\section{TORSION EXPERIMENTS}

\section{(a) Circular Cross Section}

Tinius Olsen torsion tester along with a reaction torque sensor (Figure 5) was used. The torsion tests were performed on 6061 extruded aluminum and low alloy steel 1018 bars of circular cross section ( 0.5 inch in diameter). The loading was increased and continued through the inelastic region. Figure 6 displays the data graphically and shows the torques asymptotically reach constant values for both materials with increase in twist angles. 


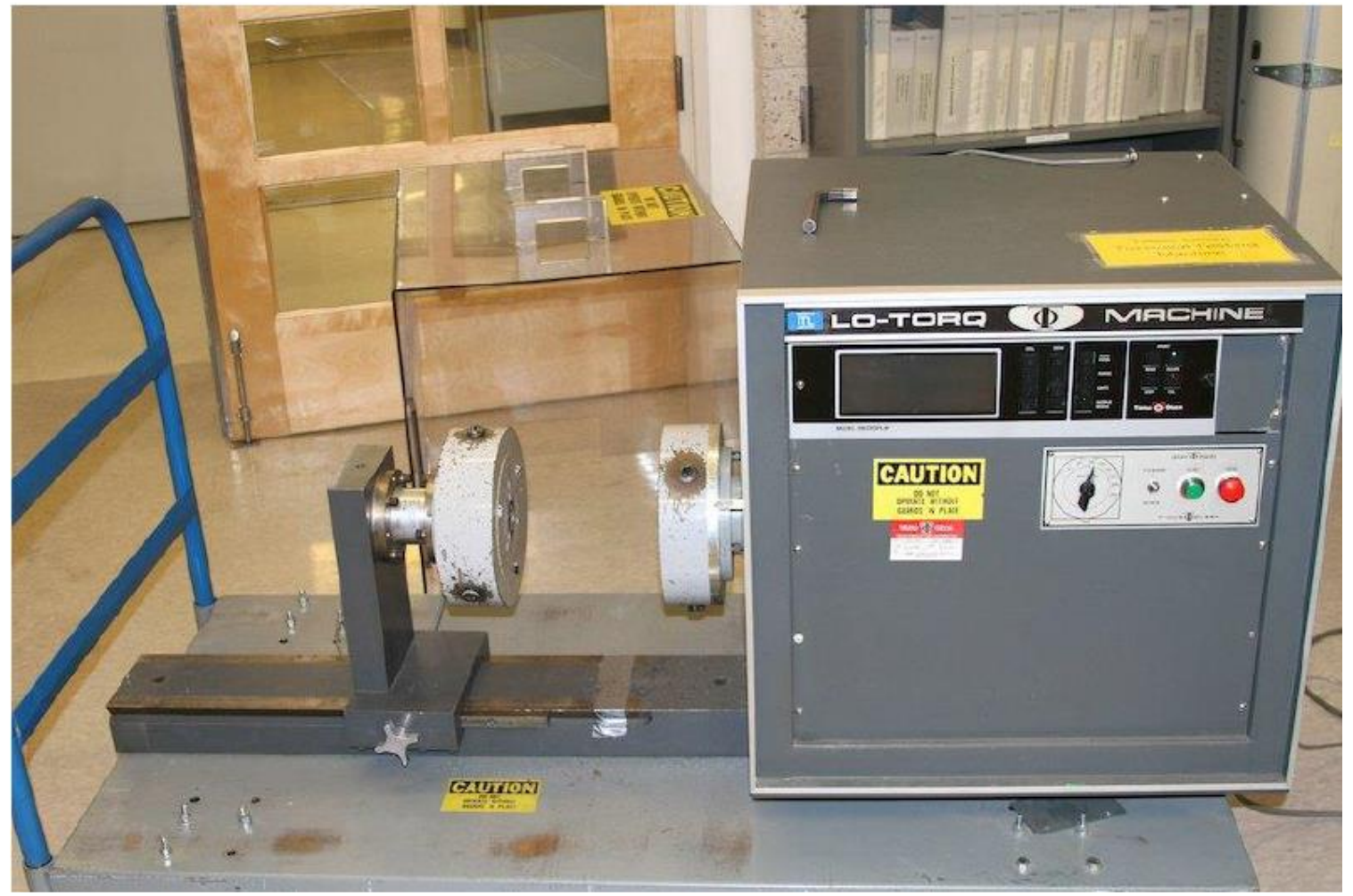

Figure 5: Experimental Setup

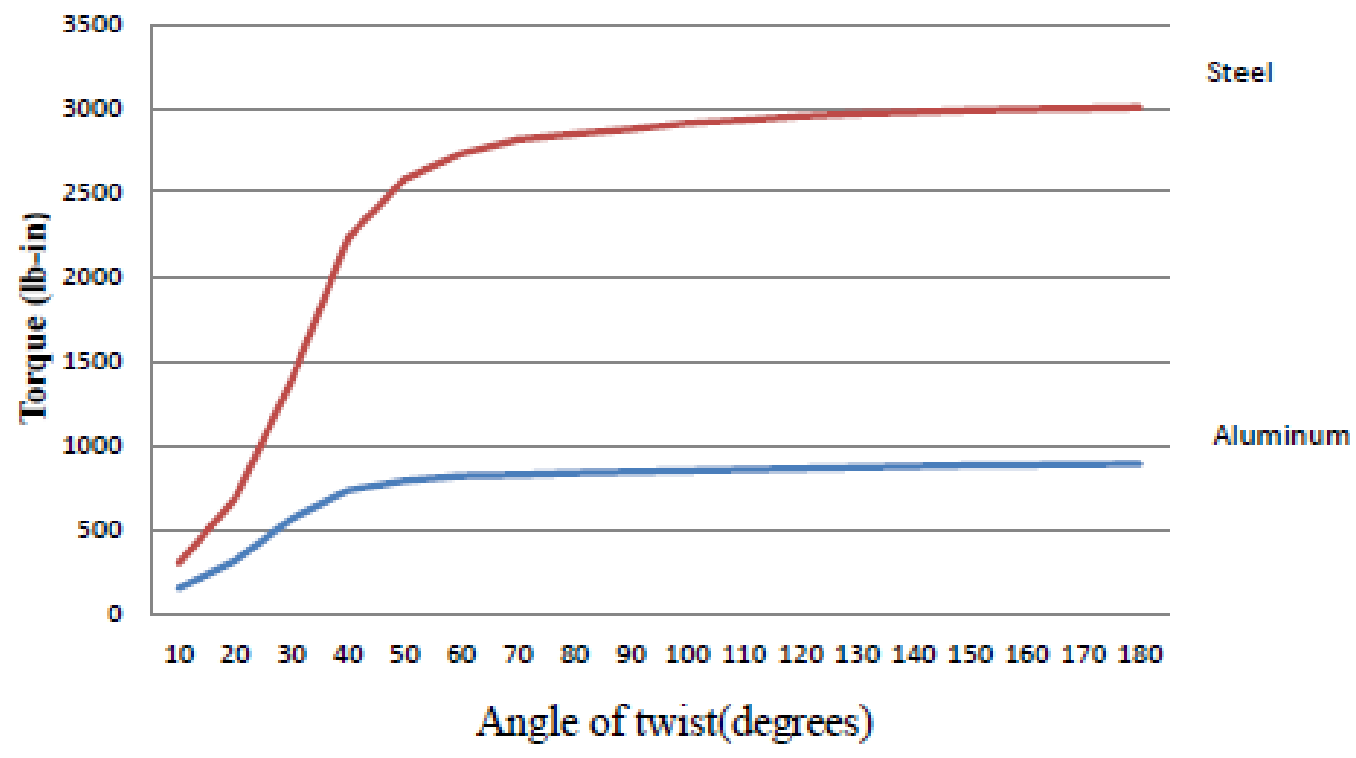

Figure 6: Torque vs. Twist angle for $1 / 2$ in. diameter aluminum and steel rods. 
It is seen from Figure 6 that for the same torque, the aluminum specimen twists considerably more that the steel specimen of the same length $(\mathrm{L})$ and cross section $(\mathrm{J})$ because of reduced shear modulus $\mathrm{G}$ of aluminum as compared to steel. The linear part of the torque-twist curves for both materials extend up to a twist angle of 20 degrees or less. This part can be used to obtain the elastic stiffness, GJ/L. ${ }^{[4]}$
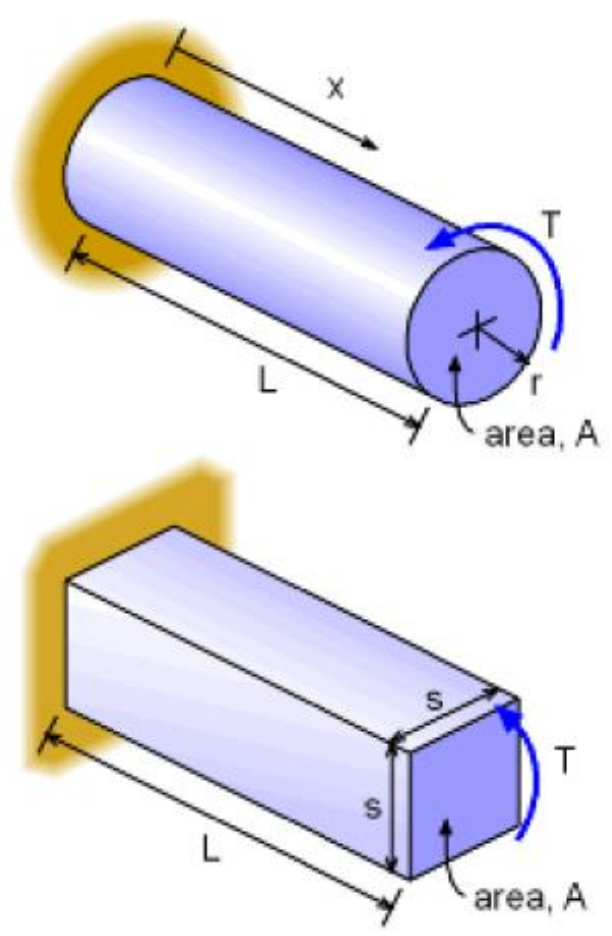

\section{Figure 7: Circular and Square Cross Sections}

Figure 7 shows a comparison of the two cross sections analyzed in this work. This section addresses the circular cross section. In the next section, the square cross section is addressed. Note that for this work, $a=s=1 / 2$ in. 
(b) Non-Circular Sections

For this purpose, specimens of a square cross section $\left(1 / 2\right.$ in $x \frac{1}{2}$ in) of aluminum 6016 -T6 were used. The torque-twist characteristics were obtained in the same way as the circular cross section, but only one material was used. The results are shown graphically in Figure 7. For the square cross section, the determination of torsional stiffness requires consideration of warping which is available only in advanced texts on Mechanics of Materials, such as, Hibbeler [6] and Seely and Smith [7].

For non-circular cross sections one needs to address warping which is assumed to vary with the rate of twist and is a function only of the position on the cross section and not on the lengthwise coordinate.

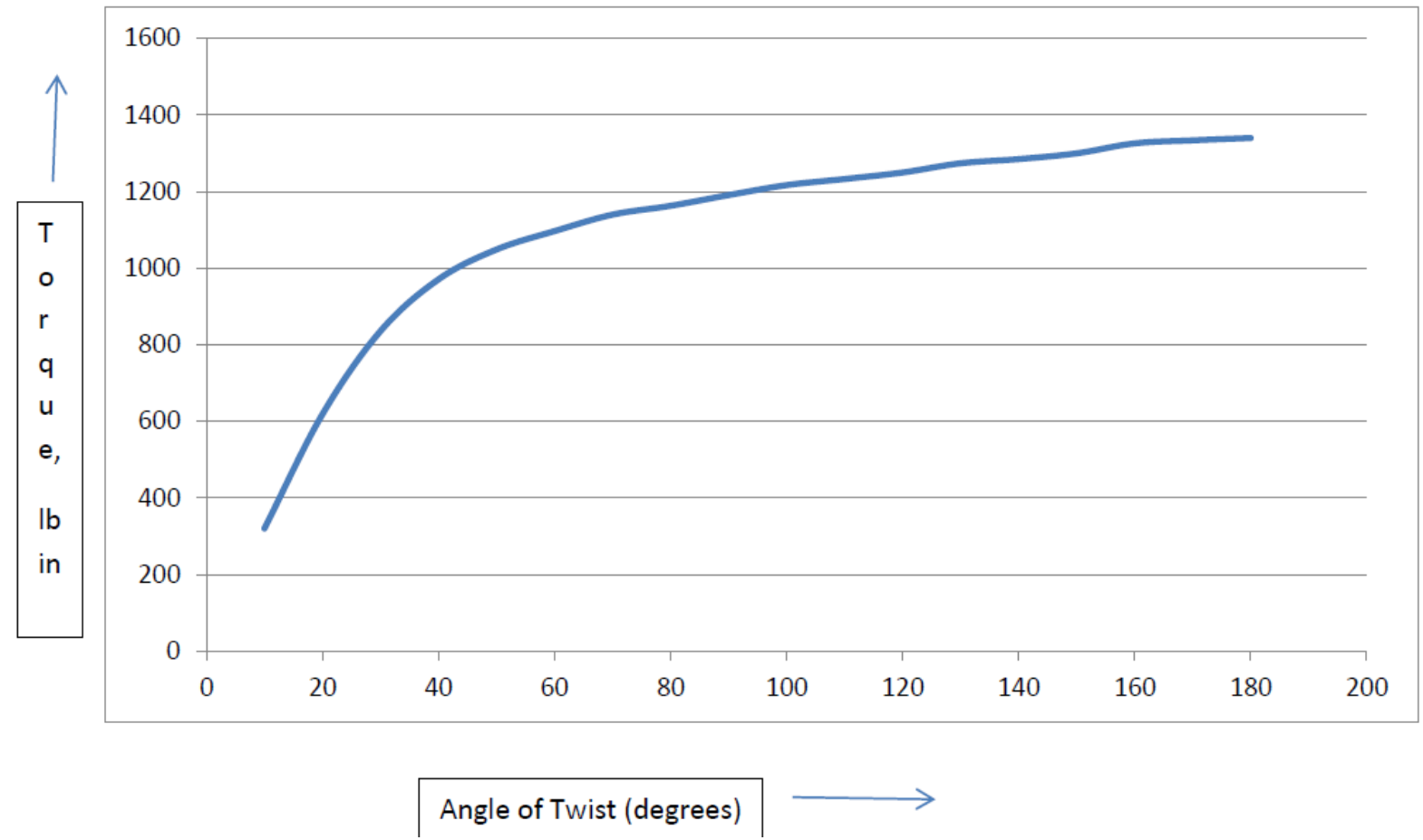

Figure 8. Torque versus Angle of Twist for Square Torsion Specimen 


\section{THEORETICAL ANALYSIS FROM SAND HEAP ANALOGY}

\section{(a) Circular Cross-Section}

The radius of the circular rod $\mathrm{a}=0.25$ in

Yield strength of aluminum (assumed), $\mathrm{k}=20,000 \mathrm{psi}$

Volume of the sand heap $=$ Twice the volume of a cone of base radius ' $a$ " and height ' $\mathrm{k}$ '

$=\mathbf{2}\left(1 / 3 \pi \mathrm{a}^{3}\right) \mathrm{k}=2 / 3 \pi(20,000)(0.25)^{3}$

$=650 \mathrm{lb}-\mathrm{in}$

The asymptotic value of the torque for the circular cross section is obtained as $888 \mathrm{in}-\mathrm{lb}$. (Figure 6 for aluminum)

\section{(b) Square Cross-Section}

Side of the square $=0.5$ in

Yield strength of aluminum (assumed), $\mathrm{k}=20,000 \mathrm{psi}$

Volume of the sand heap $=$ Twice the volume of a pyramid of side ' $a$ " and height ' $k$ '

$=\mathbf{2}\left(1 / 3 \mathrm{a}^{3}\right) \mathrm{k}=2 / 3(20,000)(0.25)^{3}$

$=1633 \mathrm{lb}$-in

The maximum value (not asymptotic) of the torque for the square cross section is obtained as 1380 in-lb. (Figure 8)

\section{EXPERIMENTS ON SAND HEAP ANALOGY AND MEMBRANE ANALOGY}

(a) Sand Heap Analogy

Circular (12-in diameter) and square (12-in to a side) trays were used on which dry uniform sand heaps were piled up. These trays were raised about 6 inches above the floor level. Following the 
work of Carey ${ }^{[4]}$ Ottawa sand produced by Ottawa Silica Company, IL was used for this purpose. The heaps were made as high as possible by pouring gentle streams of sand rolling freely down the slopes of the heap and falling off the elevated trays. The maximum sizes of the heaps once formed (cone for the circle and pyramid for the square) were weighed and volumes determined from the density of the sand $\left(1.55 \mathrm{~kg} / \mathrm{m}^{3}\right)$

Figures 9 and 10 show the heaps for the circular and square cross sections. The angles of repose were measured using an adjustable protractor. Two methods of pouring sand are shown in Figures 11 and 12.

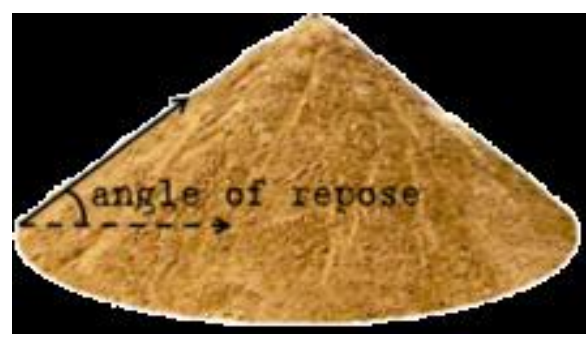

Figure 9: Sand Heap Test for Circular Cross Section

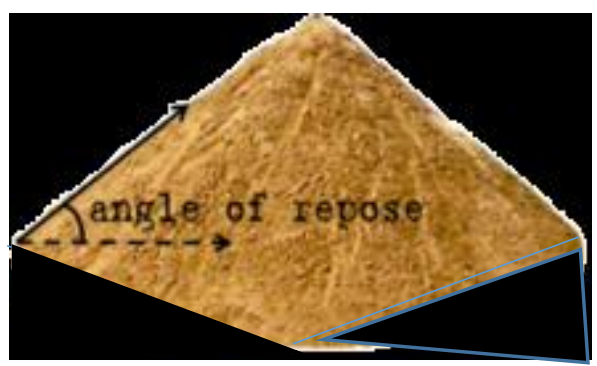

Figure 10: Sand Heap Test for Square Cross Section 

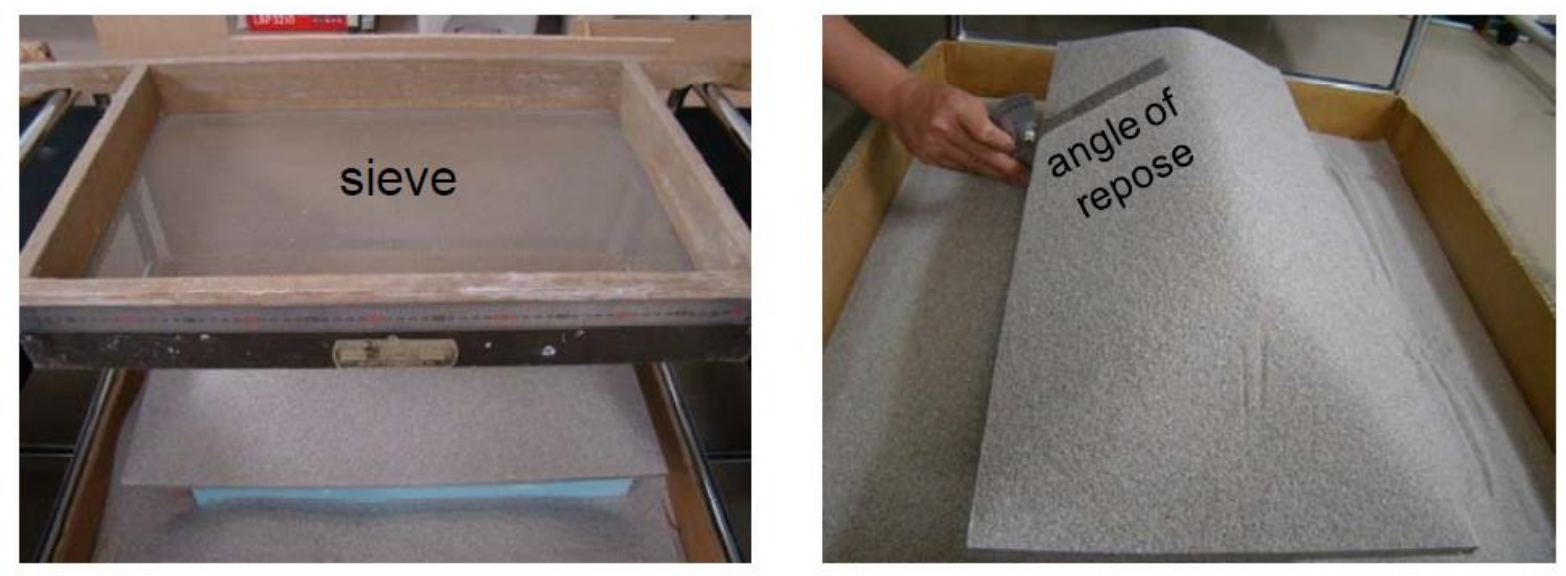

Figure 11: Using a sieve to pour sand
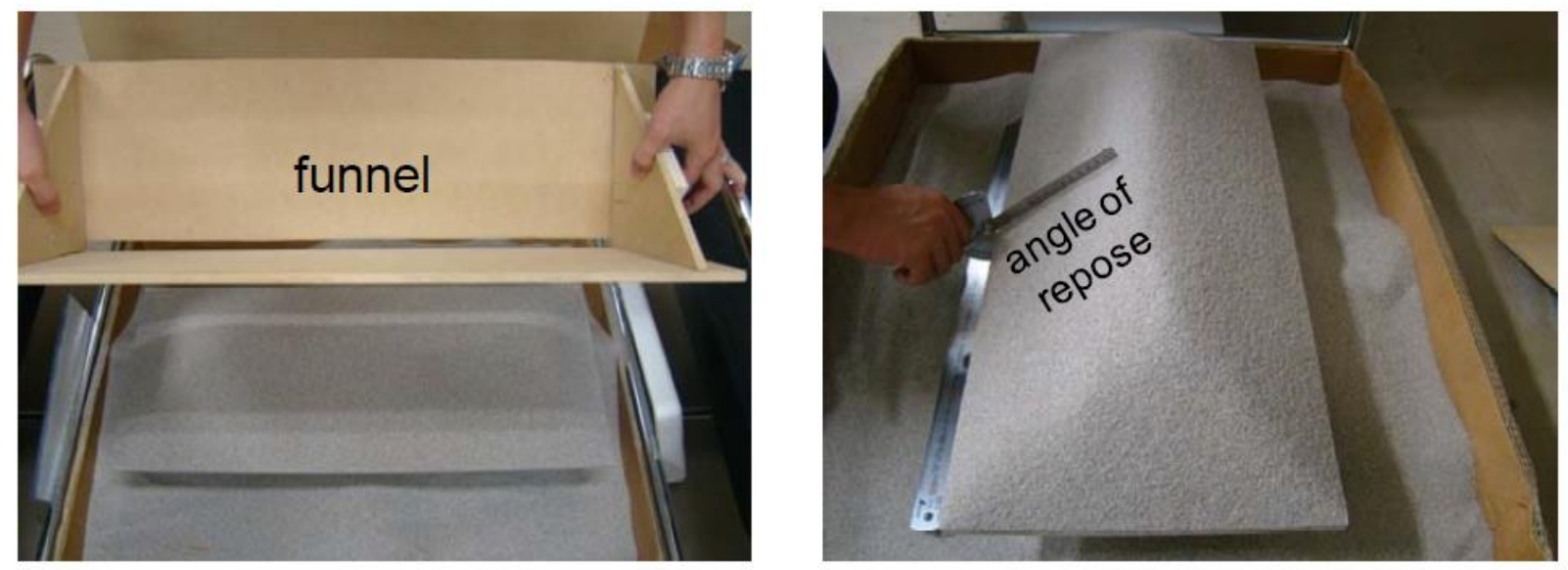

Figure 12: Using a funnel to pour sand

The experiments indicate that the final shapes of the sand heaps are independent of the sand deposition method used.

Following Nadai [5] the torque in plastic torsion is given by:

$$
T=2 \frac{k}{m} V
$$

Where $k$ is the maximum value of the shear stress in Figure 1, $m$ the maximum value of the slope of the lateral surface of the sand heap (determined experimentally as the tangent of the angle of 
repose), and $V$ is the volume of sand. The volume is calculated by weighing the sand remaining in the trays, and dividing the sand mass (in $\mathrm{kg}$ ) by the sand density given previously as 1.55 $\mathrm{kg} / \mathrm{m}^{3}$.

The experimental values using the above equation results in maximum torques within $10 \%$ of the theoretical predictions.

\section{EXPERIMENT COMBINING MEMBRANE AND SAND HEAP ANALOGIES}

An experiment has been devised in which rubber membranes are loaded by pressure from below. The membrane stretch along the outlines of a thin steel plates (cut in 12-in diameter circle and 12-in a side square) that form the tops of steel sealed boxes into which air is being pumped. The boxes have Plexiglas roofs built on their tops. The roofs have a conical shape for the circular cross-section and a pyramid shape for the square cross-section. When the intensity of the pressure becomes sufficiently high, the membrane begins to touch the roof. This corresponds to the state of initial yielding. At the limit the membrane fills the entire volume, when the fully plastic state has been attained. Figures 13 and 14 show the experimental setups for the circular cross section as well as for the square cross section.

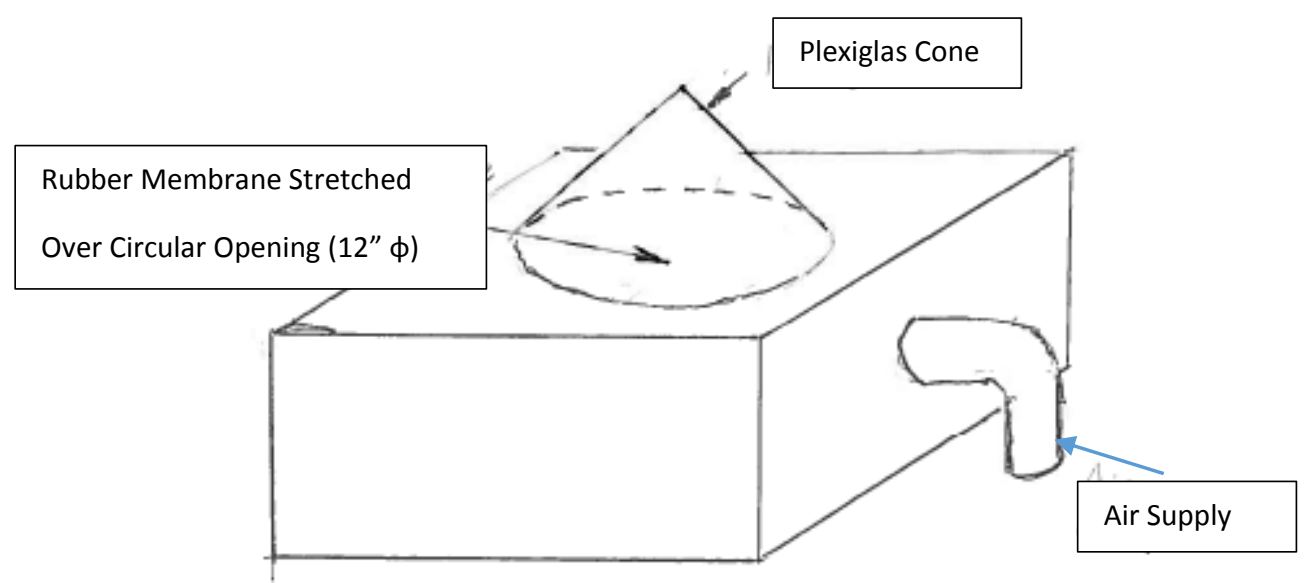

Figure 13: Analogy Test for Circular Cross-Section 


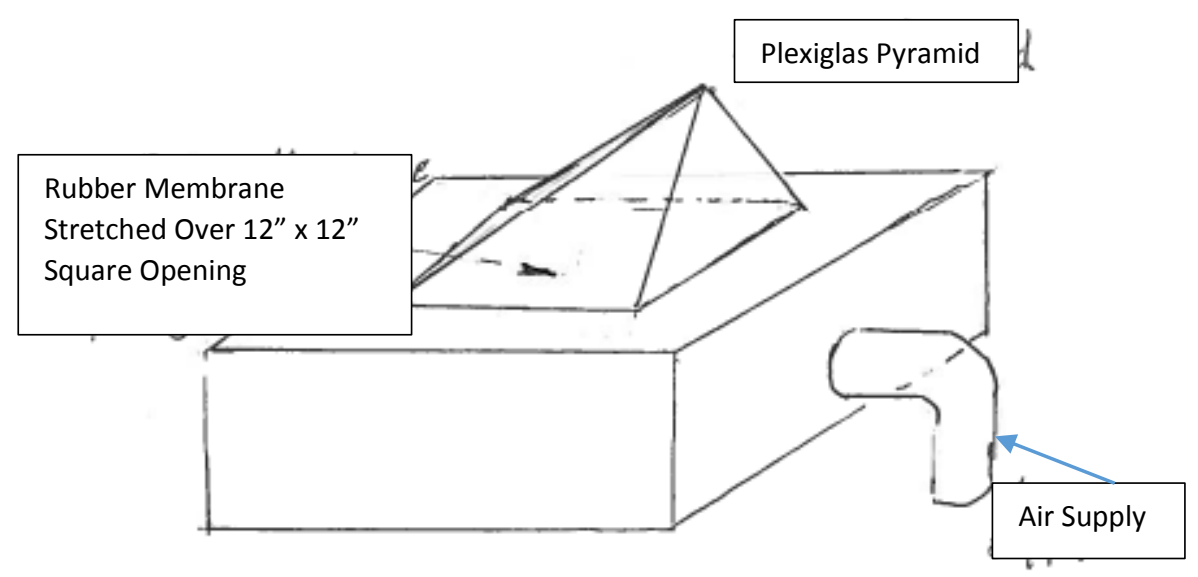

Figure 14: Analogy Test for Square Cross Section

\section{CONCLUSIONS}

The predicted maximum torque for circular cross section using sand-heap analogy is 883 in-lb versus the experimental value of $650 \mathrm{in}-\mathrm{lb}$. The predicted maximum torque for square cross section using sand-heap analogy is 1633 in-lb versus the experimental value of 1380 in-lb. The trend is reversed as we compare the results for the circular cross-section with the square cross-section. This is because there is a presence of a separate mechanism of warping in square cross-section which is absent in circular cross-section. Also the sand heap analogy is based on the assumption of zero strain hardening. As the torque twist characteristic of the square cross section indicates the presence of strain hardening we can expect discrepancies similar to what is shown in Figure 2.

As can be seen from Figure 8, a considerable amount of plastic deformation is taking place as the square aluminum rod is twisted. When the specimen is removed from the machine after a torque of $1380 \mathrm{lb}$-in was applied, it was subject to a very large plastic deformation causing it to deform permanently. 
Membrane analogy provides a mental picture of the state of stress as shown in Figure 2. The membrane for a square shaft has been shown to have the greatest slope at the midpoint of the edges and not at the corners as is sometimes supposed. In the corner the membrane has a zero slope along both edges indicating zero stress.

Experimental investigation on the torsion of square shaft provides a basis of comparison of its torsional stiffness with the analytical values that take warping into consideration. Future efforts in this area would be to measure strains using strain gages to compare with the analytical expressions for shear stresses.

\section{DISCUSSION}

This project introduces the students to material plasticity through torsion experiment involving loads that causes the material to yield. The students can appreciate how the section warps after the square section specimen is removed from the experimental setup. Because of the accompanying significant plastic deformation in the specimen, it permanently warps. For aluminum specimen, this is particularly prominent because of the high ductility of the material. In fact, it is very hard to separate the plastic deformation from the warping deformation, even for smaller values of applied torques. As the torque is increased a plastic region develops around an elastic core. There are errors introduced in the experiment primarily due to setup repeatability. 


\section{ASSESSMENT OF STUDENT LEARNING}

A number of activities in terms of solving problems, and explaining some concepts will be

(Unclassified introduced. Typical questions are:

1. Show that if the yield strength is exceeded, the governing equations for the torsional shear stress and twist do not hold. Why?

2. Calculate the maximum elastic torque when the shear yield is given for both square and circular shafts. Also calculate the maximum elastic twist for both shafts.

3. Why is the maximum torque different from the value predicted by Sand Heap Analogy?

Explain for bars of circular cross section and square cross section.

[1] Prandtl, L. Physik Z. 4, 758, 1903.

BIBLIOGRAPHY

[2] Nadai, A., Z. angnaw Math. Mech, 3, 442, 1923.

[3] Chakrabarty, J. Theory of Plasticity, $3^{\text {rd }}$ Edition, Elsevier Butterworth-Heinemann, 2006

[4] Carey, G. F., An investigation of plastic torsion in circular prismatic bars with a concentric or eccentric hole using the sand -heap analogy, MS Thesis Agricultural and Mechanical College of Texas, 1963 (Unclassified Report AD 405143, Defense Documentation Center for Scientific and Technical Information, Alexandria, VA)

[5] Nadai, A., Theory of Flow and Fracture in Solids, New York, McGraw Hill, 1950.

[6] Hibbeler, R. C., Mechanics of Materials, 8th Edition, Prentice Hall, 2011.

[7] Seely, F. B., and Smith, J. O., Advanced Mechanics of Materials, 2nd Edition, Wiley 1952 